\title{
Long term effects of non-invasive mechanical ventilation on pulmonary haemodynamics in patients with chronic respiratory failure
}

\author{
B Schönhofer, T Barchfeld, M Wenzel, D Köhler
}

\begin{abstract}
Background-It is not known whether long term nocturnal mechanical ventilation (NMV) reduces pulmonary hypertension in patients with chronic respiratory failure (CRF).

Methods-Pulmonary haemodynamics, spirometric values, and gas exchange were studied in 33 patients requiring NMV due to CRF (20 with thoracic restriction, 13 with chronic obstructive pulmonary disease (COPD)) at baseline and after 1 year of NMV given in the volume cycled mode. Patients with COPD also received supplemental oxygen.

Results-Long term NMV improved gas exchange while lung function remained unchanged. Mean pulmonary artery pressure at rest before NMV was higher in patients with thoracic restriction than in those with COPD (33 (10) $\mathrm{mm} \mathrm{Hg} v 25$ (6) $\mathrm{mm} \mathrm{Hg}$ ). After 1 year of NMV mean pulmonary artery pressure decreased in patients with thoracic restriction to 25 (6) $\mathrm{mm} \mathrm{Hg}$ (mean change $-8.5 \mathrm{~mm} \mathrm{Hg}$ $(95 \%$ CI -12.6 to -4.3$), p<0.01)$ but did not change significantly in patients with COPD (mean change $2.2 \mathrm{~mm} \mathrm{Hg}(\mathbf{9 5} \% \mathrm{CI}$ -0.3 to 4.8$)$ ).

Conclusions-Long term NMV in CRF improves pulmonary haemodynamics in patients with thoracic restriction but not in patients with COPD.

(Thorax 2001;56:524-528)
\end{abstract}

Keywords: chronic respiratory failure; chronic obstructive pulmonary disease; non-invasive mechanical ventilation; pulmonary hypertension

Krankenhaus Kloster Grafschaft, Zentrum für Pneumologie, Beatmungs- und Schlafmedizin, D-57392

Schmallenberg-

Grafschaft, Germany

B Schönhofer

T Barchfeld

$M$ Wenzel

D Köhler

Correspondence to: Dr B Schönhofer Bernd.Schoenhofer@t-online. de

Received 20 September 2000 Returned to authors 10 December 2000 Revised version received

9 January 2001

Accepted for publication

29 March 2001 on pulmonary hypertension in CRF is unknown. We therefore conducted a prospective study to investigate the long term effects of NMV on pulmonary haemodynamics after 1 year of NMV in patients with CRF secondary to both restrictive thoracic disorders and COPD.

\section{Methods}

PATIENTS

In a two year period (from 1 June 1994 to 1 June 1996) all patients who were considered for NMV were approached about the study protocol. During this period a total of 45 patients with thoracic restriction and 31 patients with COPD started NMV. Informed consent to participate in the study was given by 20 patients with thoracic restriction (group A) and 13 patients with COPD (group B). All patients finished the study.

The inclusion criteria were chronic hypercapnic respiratory failure $\left(\mathrm{PaCO}_{2}>6.4 \mathrm{kPa}\right)$ due to restrictive thoracic diseases (posttuberculosis sequelae and scoliosis, group A) or COPD (forced expiratory volume in one second $\left(\mathrm{FEV}_{1}\right)<1$ litre and $\mathrm{FEV}_{1} /$ forced vital capacity (FVC) ratio of $<50 \%$, group B). ${ }^{8}$ All patients had to have received maximal medical treatment supervised by a chest physician with no hospital admission for at least 1 month before the study and to have remained free of an exacerbation during this time. No significant difference was observed between the preadmission blood gas tensions and lung function (1 month before admission) and those obtained on admission to hospital, confirming that the patients were in a clinically stable condition.

Subjects with acute respiratory failure (requiring continuous mechanical ventilation), severe acidosis (defined as $\mathrm{pH}<7.30$ ), or left ventricular failure (defined as radiographically demonstrable congestion of pulmonary veins and/or an ejection fraction $<50 \%$ and/or left ventricular end diastolic diameter $>55 \mathrm{~mm}$ on echocardiography) were excluded. All patients underwend a polygraphic sleep study (PolyMESAM, MAP, Martinsried, Germany) before entry and those with obstructive sleep apnoea were excluded.

Anthropometric parameters, blood gas analysis, and lung function data of the two groups of patients are presented in table 1 .

\section{MEASUREMENTS}

Baseline measurements were performed before starting NMV. Spirometric tests and whole body plethysmography (Masterlab, Jaeger, 
Table 1 Anthropometric parameters, blood gas analysis, and lung function data of patients with thoracic restriction (group A) and patients with COPD (group B)

\begin{tabular}{|c|c|c|}
\hline & Group $A$ & Group B \\
\hline $\mathrm{n}$ & 20 & 13 \\
\hline Scoliosis & 11 & - \\
\hline Post-TBC & 9 & - \\
\hline COPD & - & 13 \\
\hline $\mathrm{F}: \mathrm{M}$ & $9: 11$ & $1: 12$ \\
\hline Age (years) & $63.9 \quad(8.1)$ & $50.7 \quad(8.2)$ \\
\hline $\mathrm{BMI}\left(\mathrm{kg} / \mathrm{m}^{2}\right)$ & $24.6 \quad(3.2)$ & $27.7 \quad(2.8)$ \\
\hline $\mathrm{PaO}_{2}(\mathrm{kPa})$ & $6.4 \quad(1.2)$ & $6.1 \quad(0.8)$ \\
\hline $\mathrm{PaCO}_{2}(\mathrm{kPa})$ & $7.8 \quad(1.0)$ & $8.0 \quad(1.0)$ \\
\hline $\mathrm{pH}$ & $7.35(0.04)$ & $7.36(0.04)$ \\
\hline $\mathrm{VC}(1)$ & $1.2(0.3)$ & $1.9(0.7)$ \\
\hline VC (\% pred) & $37.0 \quad(11.6)$ & $44.7 \quad(12.9)$ \\
\hline $\mathrm{FEV}_{1}(1)$ & $0.71(0.2)$ & $0.85(0.32)$ \\
\hline $\mathrm{FEV}_{1} / \mathrm{FVC}$ & $72.0 \quad(15.4)$ & 40.8 (6.8) \\
\hline
\end{tabular}

$\mathrm{BMI}=$ body mass index post-TBC $=$ post-tuberculosis seque lae; $\mathrm{PaO}_{2}, \mathrm{PaCO}_{2}=$ arterial oxygen and carbon dioxide tensions; $\mathrm{VC}=$ vital capacity; $\mathrm{FEV}_{1}=$ forced expiratory volume in one second.

Würzburg, Germany) were performed. The normal values for lung function data were those of the European Respiratory Society. ${ }^{9}$ Resting daytime arterialised capillary blood gases were measured from the hyperaemic ear lobe while breathing room air. The criterion for the effectiveness of NMV was a decrease in $\mathrm{PaCO}_{2}$ during the intervention.

Spontaneous unassisted ventilation was investigated during sleep using a digital pulse oximeter (mean and minimal oxygen saturation $\left(\mathrm{SaO}_{2}\right)$, Pulsoxy 7, AVL, Bad Homburg, Germany).

Right heart catheterisation was performed according to the technique described by Swan et $a l^{10}$ using a small diameter floated Grandjean catheter (5F, Angiomed, Germany). The catheter was introduced percutaneously into the antecubital vein. Mean pulmonary artery pressure (PAPmean), pulmonary capillary wedge pressure, and thermodilution cardiac index were recorded using a polygraph (Sirecust 1260, Siemens, Erlangen). Pulmonary vascular resistance index was calculated as PAPmean pulmonary capillary wedge pressure/cardiac index. Measurements were made at rest and during steady state exercise in the supine position. To determine the constant load, which was performed during the haemodynamic measurements, the maximal workload was defined in a pre-test protocol as the exercise intensity which the patient was able to tolerate for at least 2 minutes.

The duration of the exercise test after 1 year on NMV was identical to that of the pretreatment test. In group A the work load achieved was 39.8 (20.7) W for a period of 3.3
(1.0) minutes, while in group B the corresponding values were $51.1(20.1) \mathrm{W}$ for 3.5 (1.2) minutes.

STUDY PROTOCOL

The protocol was approved by the ethical review committee and all subjects gave their written informed consent to participate. A run in period of 1 month without NMV was used in order to confirm clinical stability as judged by gas exchange and lung function. Pre-NMV and post-NMV blood gas analysis was performed with the patient breathing room air; oxygen therapy was discontinued 1 hour before the test.

NMV was instituted using the volume cycled mode (either Dräger EV 800 (Lübeck, Germany) or PLV 100 (Respironics, Murrysville, USA)). Details on how individual ventilator settings were obtained are described elsewhere. ${ }^{11}$ Hypoxaemic COPD patients received supplemental oxygen according to the guidelines of the American Thoracic Society. ${ }^{8}$

Patients received 6-8 hours of daytime and nocturnal oxygen at a rate of $11 / \mathrm{min}$ if NMV alone did not correct the hypoxaemia. No patient with restrictive thoracic disease required oxygen therapy during the study. Pre-study medication was not changed during the study period, except for diuretic medication which was managed according to a protocol based on the clinical condition (reduced, withdrawn if permitted, or reintroduced, depending on the incidence of peripheral oedema). Follow up was scheduled every 6 weeks. All measurements were made before (baseline) and after 1 year of NMV.

\section{STATISTICAL ANALYSES}

Results are expressed as mean (SD). The Wilcoxon test was used for the intrasubject comparisons within each group. A p value of $<0.05$ was considered significant.

\section{Results}

All patients completed the pre-NMV evaluation and were started on NMV. Ventilator use per day was similar in both study groups (7.1 (0.5) hours in group A and $7.3(0.3)$ hours in group B) and of equal efficacy $\left(\mathrm{PaCO}_{2} 5.2\right.$ $(0.4) \mathrm{kPa}$ in group $\mathrm{A}, 5.4(0.4) \mathrm{kPa}$ in group B). Oxygen use averaged $15.2(0.7)$ hours/day in patients with COPD. With the additional oxygen therapy during $\mathrm{NMV}, \mathrm{PaO}_{2}$ increased significantly in these patients (from 7.3 (0.4) $\mathrm{kPa}$ to $11.7(1.0) \mathrm{kPa}$ ).

In all patients in group A diuretic medication was either reduced $(n=12)$ or discontinued

Table 2 Mean (SD) values of parameters before and after 1 year on NMV in patients with thoracic restriction (group A) and patients with COPD (group B)

\begin{tabular}{|c|c|c|c|c|c|c|}
\hline \multirow[b]{3}{*}{$\mathrm{PaO}_{2}(\mathrm{kPa})$} & \multicolumn{3}{|l|}{ Group $A$} & \multicolumn{3}{|l|}{ Group B } \\
\hline & Baseline & After 1 year $N M V$ & Mean change $(95 \%$ CI) & Baseline & After 1 year $N M V$ & Mean change $(95 \% C I)$ \\
\hline & $6.4 \quad(1.2)$ & $8.9 \quad(1.3)$ & $2.5(1.6 \text { to } 2.9)^{\star \star \star}$ & $6.1(0.8)$ & 7.2 (1.3) & $1.1(0.5 \text { to } 1.8)^{\star \star \star}$ \\
\hline $\mathrm{PaCO}_{2}(\mathrm{kPa})$ & 7.8 (1.0) & $5.9(0.6)$ & $-1.9(-2.4 \text { to }-1.3)^{\star \star \star}$ & $8.0(1.0)$ & $6.8 \quad(1.0)$ & $-1.2(-2.0 \text { to }-0.8)^{\star \star \star \star}$ \\
\hline $\mathrm{pH}$ & $7.35(0.04)$ & $7.39(0.03)$ & $0.04(0.01 \text { to } 0.05)^{\star \star \star \star}$ & $7.36(0.4)$ & $7.39(0.03)$ & $0.03(0.01 \text { to } 0.05)^{\star \star \star}$ \\
\hline $\mathrm{SaO}_{2}$ mean $(\%)$ & $72.5 \quad(9.5)$ & $90.5(5.2)$ & $18.0(12.1 \text { to } 30.4)^{\star \star \star}$ & $76.2 \quad(5.2)$ & $84.1 \quad(3.5)$ & $7.9(5.0 \text { to } 12.6)^{\star \star}$ \\
\hline $\mathrm{SaO}_{2}$ nadir $(\%)$ & $55.0 \quad(9.5)$ & $72.3(10.0)$ & $17.7(9.7 \text { to } 28.1)^{\star \star \star}$ & $60.0 \quad(5.7)$ & $68.5(7.8)$ & $8.5(3.4 \text { to } 14.1)^{\star \star}$ \\
\hline
\end{tabular}

${ }^{\star}$ Mean change in parameters $\left(95 \%\right.$ confidence interval, CI) from baseline to 1 year on NMV $\left({ }^{\star \star} \mathrm{p}<0.01,{ }^{\star \star \star} \mathrm{p}<0.001\right.$, Wilcoxon test).

$\mathrm{PaO}_{2}, \mathrm{PaCO}_{2}=$ arterial oxygen and carbon dioxide tensions; $\mathrm{SaO}_{2}=$ oxygen saturation during sleep. 
Table 3 Mean (SD) values of the haemodynamic parameters at baseline and after 1 year NMV in patients with thoracic restriction (group A) and patients with COPD (group B)

\begin{tabular}{|c|c|c|c|c|c|c|}
\hline & \multicolumn{3}{|l|}{ Group $A$} & \multicolumn{3}{|l|}{ Group B } \\
\hline & Before exercise & $p$ value & $\operatorname{Max}$ & Before exercise & $p$ value & $\operatorname{Max}$ \\
\hline \multicolumn{7}{|l|}{ Baseline } \\
\hline PAPmean (mm Hg) & $33.2(10.0)$ & \#\#\# & $55.1(14.4)$ & $25.3(6.0)$ & \#\#\# & $44.8(14.1)$ \\
\hline PCW (mm Hg) & $9.0(5.0)$ & \#\#\# & $16.4(5.5)$ & $8.7(3.2)$ & \#\#\# & $15.5(4.6)$ \\
\hline PVRI $\left(\right.$ dyn $\left./ \mathrm{s} / \mathrm{cm}^{5} / \mathrm{m}^{2}\right)$ & $606(200)$ & & $654(172)$ & $410(127)$ & $\#$ & $474(143)$ \\
\hline \multicolumn{7}{|l|}{ After 1 year NMV } \\
\hline PAPmean (mm Hg) & $24.8(6.2)$ & \#\#\# & $46.9(13.9)$ & $27.5(6.0)$ & \#\#\# & $47.8(10.2)$ \\
\hline $\mathrm{PCW}(\mathrm{mm} \mathrm{Hg})$ & $9.2(3.4)$ & \#\#\# & $16.4(5.5)$ & $9.2(2.5)$ & \#\#\# & $16.5(5.0)$ \\
\hline PVRI $\left(\mathrm{dyn} / \mathrm{s} / \mathrm{cm}^{5} / \mathrm{m}^{2}\right)$ & $432(158)$ & \# & $508(169)$ & $408(92)$ & \# & $471(111)$ \\
\hline \multicolumn{7}{|l|}{ Mean change $(95 \% \mathrm{CI})$} \\
\hline PAPmean $(\mathrm{mm} \mathrm{Hg})$ & $-8.5(-12.6 \text { to }-4.3)^{\star \star}$ & & $-8.2(-14.6 \text { to }-1.7)^{\star}$ & $2.2(-0.3$ to 4.8$)$ & & $3.0(-2.6$ to 8.6$)$ \\
\hline $\mathrm{PCW}(\mathrm{mm} \mathrm{Hg})$ & $0.2(-2.3$ to 2.7$)$ & & $-0.1(-4.6$ to 4.3$)$ & $0.5(-0.9$ to 2.0$)$ & & $1.0(-0.8$ to 2.8$)$ \\
\hline PVRI $\left(\mathrm{dyn} / \mathrm{s} / \mathrm{cm}^{5} / \mathrm{m}^{2}\right)$ & $-174(-288 \text { to }-61)^{\star \star}$ & & $-146(-239 \text { to }-53)^{\star}$ & $-2(-99$ to 95$)$ & & $-3(-46$ to 40$)$ \\
\hline
\end{tabular}

PAP = pulmonary arterial pressure; PCW = pulmonary wedge pressure; PVRI = pulmonary vascular resistance index.

${ }^{\star}$ Mean change in haemodynamic parameters $\left(95 \%\right.$ confidence interval, CI) from baseline to 1 year NMV ( ${ }^{\star} \mathrm{p}<0.05,{ }^{\star} \mathrm{k} p<0.01$, Wilcoxon test). \#Comparison of the values before and at the end of maximal load $(\max )(\# \mathrm{p}<0.05, \# \# \# \mathrm{p}<0.001$, Wilcoxon test).
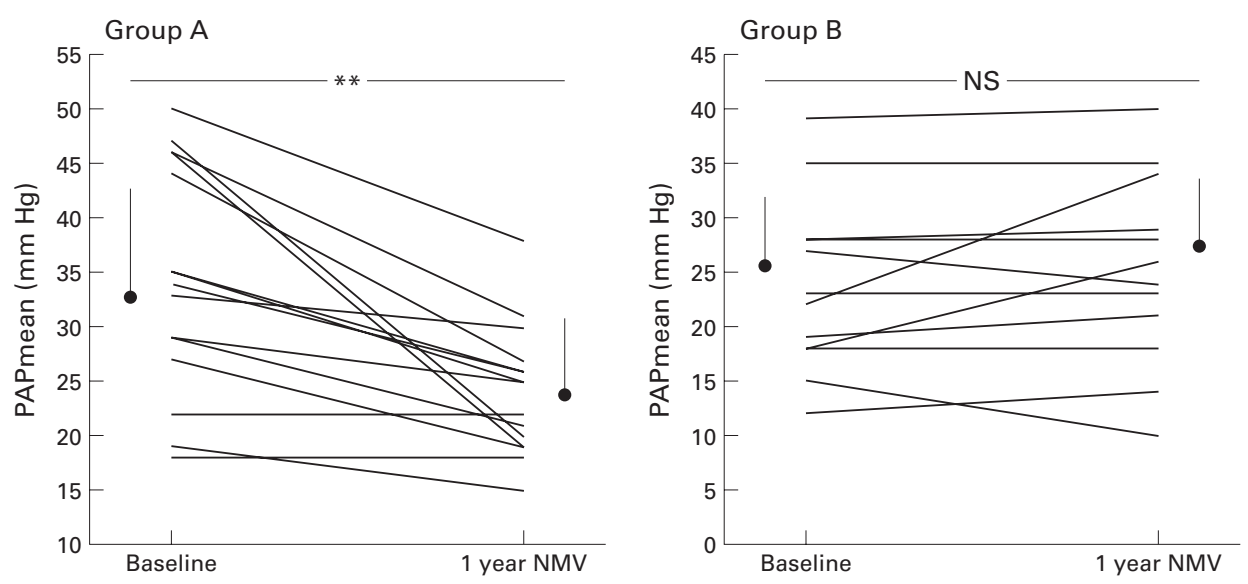

Figure 1 Individual values of mean pulmonary artery pressure (PAPmean) before and after 1 year of NMV in patients with thoracic restriction (group $A$ ) and patients with COPD (group B). ${ }^{\star *} p<0.01$ (Wilcoxon).

$(\mathrm{n}=8)$. In group $\mathrm{B}$ diuretic medication was reduced in two patients, continued in six, and five patients did not receive diuretic medication.

Gas exchange data at baseline and after 1 year of NMV are summarised in table 2. After 1 year of NMV both groups showed significant improvement in daytime blood gas tensions and in nocturnal $\mathrm{SaO}_{2}$ measured during unassisted ventilation on room air. However, the increase in $\mathrm{PaO}_{2}, \mathrm{SaO}_{2}$ mean and nadir, and decrease in $\mathrm{PaCO}_{2}$ were lower in group $\mathrm{B}$ than in group A (table 2). Lung function remained unchanged in both groups.

Baseline PAPmean and pulmonary vascular resistance index at rest were moderately increased in group A and only mildly increased in group B (table 3). Pulmonary hypertension was more severe in group $\mathrm{A}$ than in group $\mathrm{B}$ and worsened during exercise in both groups (table 3 ). Normal pulmonary capillary wedge pressures at rest and at the end of the exercise excluded left heart dysfunction in both groups.

After 1 year of NMV resting PAPmean (table 3, fig 1) and pulmonary vascular resistance index decreased significantly in group A (table 3). In contrast, PAPmean (fig 1) and pulmonary vascular resistance index at rest were unchanged in patients in group B (table 3 ). In both groups capillary wedge pressure and cardiac index did not change. Compared with the
pre-NMV data, the degree of exercise induced worsening of pulmonary hypertension after 1 year of NMV was similar in both groups.

\section{Discussion}

After 1 year of NMV a substantial reduction in PAPmean was observed in patients in group A. This reduction may contribute to the benefit conferred by NMV in patients with CRF due to thoracic restriction. ${ }^{1}$

Pulmonary hypertension at baseline was more severe in group A patients than in those in group $\mathrm{B}$, despite comparable degrees of hypercapnia and hypoxaemia. Furthermore, pulmonary hypertension did not improve in patients with COPD after 1 year of NMV.

This study has three limitations. Firstly, the ideal study design would have been a randomised controlled trial to determine whether NMV was the important factor underlying the findings. We used a prospective cohort study design as a randomised controlled trial would have been unethical in patients with thoracic restriction as there is already evidence of a marked reduction in pulmonary hypertension due to NMV.

Secondly, the number of recruited patients was relatively small, despite a 3 year study period. Between 1 June 1994 and 1 June 1996 all patients with COPD and with thoracic restriction who began NMV in our hospital 
were approached to participate in the study; $41.9 \%$ of patients with COPD and $44.4 \%$ with thoracic restriction consented to the study protocol. However, we believe that the findings can be generalised, particularly with respect to patients with thoracic restriction. Furthermore, the fact that our findings are based on right heart catheterisation, the gold standard of haemodynamic measurements, may compensate for the relatively small size of the study sample.

A third limitation is the fact that the patients with COPD had a lower pulmonary artery pressure at baseline than the patients with thoracic restriction, despite similar baseline blood gas abnormalities without oxygen supplementation during the day and at night in both groups. Compared with patients with thoracic restriction, relatively few COPD patients had significant pulmonary hypertension. This is important since there is less room for improvement in pulmonary hypertension in a patient group that started out with lower pressures. We therefore did not compare the haemodynamic findings between the two groups.

Hypoxaemia and hypercapnia both increase pulmonary vasculature tone and induce pulmonary hypertension. ${ }^{12-16}$ In our study NMV led to a sustained improvement in gas exchange, shown as a decrease in $\mathrm{PaCO}_{2}$ during daytime spontaneous ventilation and an increase in $\mathrm{SaO}_{2}$ during the one night sleep study without NMV. Our findings support the hypothesis that the increase in $\mathrm{PaO}_{2}$ and decrease in $\mathrm{PaCO}_{2}$ caused by NMV are instrumental in causing the improvement in pulmonary haemodynamics observed in patients with CRF due to thoracic restriction. In contrast, despite equal efficacy of NMV on ventilation after 1 year, pulmonary hypertension at rest was unchanged and hypercapnia and hypoxaemia during spontaneous breathing without oxygen supplementation were reduced but persisted in patients with COPD. Despite additional oxygen therapy (mean 15.2 hours/day), improvement in gas exchange in the COPD group was less than in patients with thoracic restriction. The oxygen therapy free interval during the day in the COPD group (lasting approximately 9 hours/ day) may be an additional cause of the hypoxaemia induced pulmonary hypertension.

Finally, mechanical factors may also have an important effect on the pulmonary circulation. Wilkinson et al found that longitudinal muscularisation of larger vessels resulted from mechanical distortion (stretching) in the lungs caused by progressive airway obstruction. ${ }^{17}$ This possible cause of pulmonary hypertension in COPD would not be expected to be changed by NMV.

It is interesting to speculate why peripheral oedema often remained unchanged and diuretics were still indicated in patients in group B. Apart from right heart function, peripheral oedema is related to renal and endocrine factors. ${ }^{18}$ Furthermore, a persistent hypoxaemia during the interval without oxygen supplementation and the higher $\mathrm{CO}_{2}$ during spontaneous breathing in patients in group $\mathrm{B}$ may have important effects on renal blood flow. ${ }^{15}$
As there are no comparable data available on the long term effects of NMV on pulmonary hypertension in COPD, it is of interest to consider two previous studies which have investigated the influence of long term oxygen therapy on pulmonary hypertension in COPD. ${ }^{19}{ }^{20}$ In 21 patients receiving at least 15 hours oxygen/day for 2 years the PAPmean did not change while in 21 patients not treated with long term oxygen therapy the PAPmean increased by $2.8 \mathrm{~mm} \mathrm{Hg}$ per year. ${ }^{19}$ In patients treated with oxygen for $>18$ hours/day the PAPmean fell by $3 \mathrm{~mm} \mathrm{Hg}$, whereas no change was reported in patients receiving oxygen for 12 hours/day. ${ }^{20}$ Further uncontrolled studies found a small decrease in pulmonary hypertension in patients receiving oxygen for 14-16 hours/day. ${ }^{21} 22$

Preliminary data from the French and Italian multicentre trials failed to show a significant benefit of NMV in COPD compared with long term oxygen therapy. ${ }^{56}$ However, in an uncontrolled study Léger $e t$ al showed that NMV may reduce the frequency of hospital admissions due to acute exacerbations in COPD. ${ }^{1}$ In this context, Kessler et al found that baseline hypercapnia and pulmonary hypertension, even if moderate, are independent predictors of hospitalisation due to acute exacerbations of COPD. ${ }^{7}$ In conjunction with our findings concerning pulmonary haemodynamics, most patients with CRF due to COPD may not benefit from NMV. However, further studies are needed to elucidate predictive parameters for the subgroup of patients with COPD who respond to NMV.

There are few published data on pulmonary haemodynamics before and during the follow up of NMV in patients with CRF, but data have recently become available on the short term haemodynamic effects of NMV in CRF in a heterogeneous population. ${ }^{23}$ Using non-invasive methods (echocardiography and radionuclide assessments), Thorens et al showed that mean and systolic PAP decreased and right ventricular ejection fraction increased after 4 hours and after a few days of NMV in patients with $\mathrm{CRF}^{23}$ However, compared with right heart catheterisation, non-invasive measurements of pulmonary haemodynamics may be considered less reliable. To the best of our knowledge, our study is the first to address directly the long term changes in pulmonary haemodynamics due to NMV using pulmonary catheterisation in non-invasively ventilated patients with CRF secondary to different diseases.

In conclusion, NMV caused a substantial reduction in pulmonary hypertension after 1 year of treatment in patients with CRF due to restrictive lung and thoracic diseases. In contrast, NMV had no effect on pulmonary hypertension in patients with CRF resulting from COPD. This differential effect on pulmonary haemodynamics may account for the relatively greater benefit conferred by NMV in patients with restrictive lung and thoracic diseases than in those with COPD. The lack of effect of NMV on pulmonary hypertension in COPD underlines the need for further studies to identify subgroups responding to this intervention. 
The authors thank $M$ Polkey and $M$ Pfeifer for their critical remarks and R Ragette for the linguistic advice on the article.

The data in this study have already been presented at a workshop of the German study group on Home and Long Term Mechanical Ventilation held on 26-28 March 1998 and published as a non-peer reviewed paper in a Supplement of Pneumologie (Schönhofer B, Barchfeld T, Wenzel M, Köhler D. Auswirkungen der intermittierenden Selbstbeatmung auf die pulmonale Hypertonie bei chronisch ventilatorischer Insuffizienz. Pneumologie 1999;53:S113-5).

1 Léger P, Bedicam JM, Cornette A, et al. Nasal intermittent positive pressure ventilation: long-term follow-up in patients with severe chronic respiratory insufficiency. Chest 1994;105:100-5.

2 Simonds AK, Elliott MW. Outcome of domiciliary nasal intermittent positive pressure ventilation in restrictive and obstructive disorders. Thorax 1995;50:604-9.

3 Hill NS. Noninvasive ventilation. Am Rev Respir Dis 1993;147:1050-5.

4 Meyer TJ, Hill NS. Noninvasive positive pressure ventilation to treat respiratory failure. Ann Intern Med 1994;120: $760-70$.

5 Clini F, Sturani C. The Italian multicentric study of non-invasive nocturnal pressure support ventilation (NPSV) in COPD patients. Am $\mathcal{F}$ Respir Crit Care Med 1999;159:A295.

6 Muir JF, De La Salmoniere P, Curvelier A, et al. Survival of severe hypercapnic COPD under long-term home mechanical ventilation with NPPV + oxygen versus oxygen therapy alone. Preliminary results of a European multicentre study. Am f Respir Crit Care Med 1999;159:A295.

7 Kessler R, Faller M, Fourgaut G, et al. Predictive factors of hospitalization for acute exacerbation in a series of 64 patients with chronic obstructive pulm in Respir Crit Care Med 1999;159:158-64.

8 American Thoracic Society. Standards for the diagnosis and care of patients with chronic obstructive pulmonary
disease. Am F Respir Crit Care Med 1995;152:77-121.

9 Quanjer PH, Dalhuijsen A, Van Zomeren BC. Summary equations of reference values. Bull Eur Physiopathol Respir 1983;19(Suppl 5):45-51.
10 Swan HJC, Ganz W, Forrester JS, et al. Catheterisation of the heart in man with use of a flow directed catheter. N Engl 7 Med 1970;283:447-51.

11 Schönhofer B, Sonneborn M, Haidl P, et al. Comparison of two different modes for noninvasive mechanical ventilation in chronic respiratory failure: volume versus pressure controlled device. Eur Respir 7 1997;10:184-91.

12 Hasleton PS, Health D, Brewer DB. Hypertensive pulmonary vascular disease in states of chronic hypoxia. $\mathcal{F}$ Pathol 1968;95:431-40

13 Kiely DG, Cargill IC, Lipworth J. Effects of hypercapnia on hemodynamic, inotropic, lusitropic, and electrophysiologic indices in humans. Chest 1996;109:1215-21.

14 Meyrick B, Reid L. The effect of hypoxia on rat pulmonary arterial circulation: an ultrastructural study. Lab Invest 1978;38:188-200

15 Sharkey RA, Mulloy EM, O'Neill SJ. The acute effects of oxygen and carbon dioxide on renal vascular resistance in patients with an acute exacerbation of COPD. Chest 1999; 115:1588-92.

16 Viitanen A, Salmenperä M, Heinonen J. Right ventricular esponse to hypercapnia after cardiac surgery. Anaesthesiology 1990;73:393-400.

17 Wilkinson $\mathrm{M}$, Langhome CA, Heath $\mathrm{D}$, et al. A pathophysiological study of 10 cases of hypoxic cor pulmonale. $Q \mathcal{F}$ Med 1988;66:65-85.

18 Baudouin SV. Oedema and cor pulmonale revisited. Thorax 1997;52:401-2.

19 MRC Working Party. Report of British Medical Research Council Working Party. Long term oxygen therapy in chronic bronchitis and emphysema. Lancet 1981;i: 681-6.

20 Nocturnal Oxygen Therapy Trial Group. Continuous or nocturnal oxygen therapy in hypoxaemic COPD. Ann Intern Med 1980;93:391-8.

21 Cooper CB, Waterhouse J, Howard P. Twelve year clinical study of patients with hypoxic cor pulmonale given long-term domiciliary oxygen therapy. Thorax 1987;42: $105-10$

22 Zielinski J, Tobiasz M, Hawrylkiewicz I, et al. Effects of long-term oxygen therapy on pulmonary hemodynamics in COPD patients. Chest 1998;113:65-70.

23 Thorens JB, Ritz M, Reynard C, et al. Haemodynamic and endocrinological effects of noninvasive mechanical ventilaendocrinological effects of noninvasive mechanical ventila-
tion in respiratory failure. Eur Respir f 1997;10:2553-9. 